\title{
Leptin enhances wound re-epithelialization and constitutes a direct function of leptin in skin repair
}

\author{
Stefan Frank, Birgit Stallmeyer, Heiko Kämpfer, Nicole Kolb, and Josef Pfeilschifter
}

Zentrum der Pharmakologie, Klinikum der Johann Wolfgang Goethe-Universität, Frankfurt, Germany

Address correspondence to: Stefan Frank, Institut für Allgemeine Pharmakologie und Toxikologie,

Klinikum der Johann Wolfgang Goethe-Universität Frankfurt/Main, Theodor-Stern-Kai 7, D-60590 Frankfurt/Main,

Germany. Phone: 69-6301-6954; Fax: 69-6301-7942; E-mail: S.Frank@em.uni-frankfurt.de.

Stefan Frank and Birgit Stallmeyer contributed equally to this work.

Received for publication December 14, 1999, and accepted in revised form July 11, 2000.

\begin{abstract}
Wound-healing disorders are a therapeutic problem of extensive clinical importance. Leptin-deficient $o b / o b$ mice are characterized by a severely delayed wound healing that has been explained by the mild diabetic phenotype of these animals. Here we demonstrate that systemically and topically supplemented leptin improved re-epithelialization of wounds in $o b / o b$ mice. Leptin completely reversed the atrophied morphology of the migrating epithelial tongue observed at the wound margins of leptin-deficient animals into a well-organized hyperproliferative epithelium. Moreover, topically supplemented leptin accelerated normal wound-healing conditions in wild-type mice. As assessed by immunohistochemistry, proliferating keratinocytes located at the wound margins specifically expressed the leptin-receptor subtype $\mathrm{ObRb}$ during repair. Additionally, leptin mediated a mitogenic stimulus to the human keratinocyte cell line $\mathrm{HaCaT}$ and human primary keratinocytes in vitro. Therefore, leptin might represent an effective novel therapeutic factor to improve impaired wound-healing conditions.
\end{abstract}

J. Clin. Invest. 106:501-509 (2000)

\section{Introduction}

Leptin, the $o b$ gene product, has been characterized as a satiety-regulating cytokine that is predominantly expressed by adipocytes and secreted into the bloodstream $(1,2)$. Obese/obese $(o b / o b)$ mice, homozygous for a spontaneous mutation in the $o b$ gene, fail to produce leptin and exhibit severe obesity. The appetite-regulating effect of leptin has been shown to be dependent on binding of leptin to the corresponding leptin-receptor subtype ObRb in the hypothalamic region. Subsequently, activation of ObRb leads to initiation of the Janus kinase-signal transducers (Jak-signal transducers) and activators of transcription-signaling cascade (STAT), which finally results in increased lipolytic activity and a decrease of food intake (3-5). However, the leptin-receptor splice variant ObRb was shown to be expressed in various tissues in rodents, including lung, pancreatic beta islets, and kidney (6), suggesting that leptin additionally mediates extrahypothalamic actions and, thus, might exert diverse biological functions (7, 8). Accordingly, leptin triggers reproductive functions in vivo $(9,10)$ and serves as a mitogen for a growing number of cell types, including endothelial cells, monocytes, lung epithelial cells, and pancreatic beta cells in vitro (11-15). Moreover, antiapoptotic activities of leptin have been shown for myeloid leukemia cells (16). In addition, leptin has been discussed to play an important role in angiogenesis, because leptin promotes the formation of new blood vessels $(11,17)$.
For many years, leptin-deficient $o b / o b$ mice have been used as a model system to analyze molecular characteristics of impaired wound healing. The severe wound-healing difficulties observed in $o b / o b$ mice have been explained by the diabetic phenotype of the animals. However, growth factors and cytokines are central to a normal wound-healing process (18). Thus, EGF and keratinocyte growth factor (KGF) are wellknown to be potent mitogens for epithelial cells and, therefore, essentially involved in re-epithelialization during wound healing $(19,20)$. The observation that leptin mediates angiogenic and mitogenic effects in vitro further implicates an important role for leptin as a mitogenic factor during tissue regeneration in vivo. Here we demonstrate that leptin markedly improved re-epithelialization of excisional wounds in $o b / o b$ mice and accelerated normal wound-healing conditions in wild-type mice. Furthermore, our studies demonstrate that leptin acts as potent mitogenic stimulus to keratinocytes during skin repair. Thus, our data clearly suggest that the delayed wound healing observed in $o b / o b$ mice might therefore be due, at least partially, to impaired re-epithelialization processes in the absence of the growth factor leptin during cutaneous repair.

\section{Methods}

Animals. Female C57BL/6J-ob/ob and wild-type mice (Balb/c) were obtained from The Jackson Laboratory (Bar Harbor, Maine, USA) or Charles River (Sulzfeld, Germany) and maintained under a 12-hour-light/12- 
hour-dark cycle at $22^{\circ} \mathrm{C}$ until they were 8 weeks of age. At this time they were caged individually, monitored for body weight, and wounded as described below.

Leptin treatment of mice. To investigate the function of systemically applied leptin during the wound-healing period, female C57BL/6J-ob/ob mice were injected intraperitoneally once a day at 8 am with murine recombinant leptin $(5 \mu \mathrm{g} / \mathrm{g}$ body weight $)$ in $0.5 \mathrm{~mL}$ PBS per injection for 13 days. To investigate the effect of locally applied leptin on the wound-healing process, wounds of female C57BL/6J-ob/ob mice were covered with $1 \mu \mathrm{g}$ leptin in $20 \mu \mathrm{L}$ PBS twice a day (8 am, $8 \mathrm{pm})$. Wounds of wild-type mice $(\mathrm{Balb} / \mathrm{c})$ received $5 \mu \mathrm{g}$ leptin in $20 \mu \mathrm{L}$ PBS twice a day $(8 \mathrm{am}, 8$ pm). Control mice were treated with PBS alone. Wounds from "mixed" mice were treated with topically applied leptin on the left side of the backs and with PBS alone on the right side of the backs, respectively. Murine recombinant leptin was from R\&D Systems (Wiesbaden, Germany).

Wounding and preparation of wound tissues. To examine leptin functions on the wound-healing process, six full-thickness wounds were created on each animal, and skin biopsy specimens from four animals were obtained 1, 3, 5, 7, and 13 days after injury, as described recently (21). For leptin-, or PBS-treated $o b / o b$ mice, body weight and blood glucose levels (Accutrend sensor; Boehringer Mannheim, Mannheim, Germany) were determined. For every experimental time point, the wounds from three animals and the nonwounded back skin from three animals were combined and used for RNA ( $n=9$ wounds) and protein isolation ( $n=6$ wounds), respectively. All animal experiments were carried out according to the guidelines and with the permission of the local government of Hessen, Germany.

$R N A$ isolation and RNase protection analysis. RNA isolation was performed as described (22). Twenty micrograms of total RNA from wounded or nonwounded skin were used for RNase protection assays. RNase protection assays were carried out as described previously (23), with at least two different sets of RNA from independent wound-healing experiments. A 366-bp fragment corresponding to nucleotides 506-871 of the murine leptin-receptor cDNA ObRb (24) was used as a template. RNases A and T1 were from Roche Biochemicals (Mannheim, Germany).

Western blot analysis. Skin lysates were prepared as described previously $(21)$. Total protein $(50 \mu \mathrm{g})$ from unwounded back skin and 1-day, 3-day, 5-day, 7-day, and 13-day wounds of wild-type mice (Balb/c) or $o b / o b$ mice treated with either PBS or leptin were separated using SDS gel-electrophoresis. After transfer to a PVDF membrane, leptin receptor ObRa- and ObRb-specific proteins were detected using a polyclonal antiserum directed against a synthetic peptide whose sequence is derived from amino acids 577-594 of the mouse leptin receptor (ABR, Golden, Colorado, USA). A secondary Ab coupled to horseradish peroxidase (Biomol, Hamburg,
Germany) and the enhanced chemiluminescence detection system (Amersham Pharmacia Biotech, Freiburg, Germany) were used to visualize leptin-receptor protein.

Histological analysis. Mice were wounded as described above. At days 3, 5, 7, and 13 after wounding mice were sacrificed, and complete wounds were isolated from the middle of the back, bisected, and frozen in tissue-freezing medium. Immunohistochemistry was carried out on 6$\mu \mathrm{m}$ frozen sections as described previously (21). Sections were subsequently incubated for 60 minutes at room temperature with a polyclonal antiserum recognizing the $\mathrm{ObRa}$ and $\mathrm{ObRb}$ form of the murine leptin receptor $(\mathrm{ABR})$, a polyclonal $\mathrm{Ab}$ raised against the $\mathrm{COOH}$-terminus of the murine ObRb leptin-receptor subtype (Santa Cruz Biotechnology Inc., Heidelberg, Germany) or a polyclonal antiserum raised against murine Ki67 (Dianova, Hamburg, Germany) diluted 1:100 in PBS, 0.1\% BSA.

Cell culture of buman primary keratinocytes. Human primary epidermal keratinocytes were purchased from BioWhittaker Europe Inc. (Verviers, Belgium). Cells were cultured in a defined keratinocyte medium (KBM2) according to the instructions of the supplier.

5-bromo-2'-deoxyuridine assay. Keratinocytes (HaCaT cell line [25] and human primary cells) were seeded on 96-well plates in a concentration of $5 \times 10^{2}$ cells per well. After 24 hours of incubation, cells were subsequently stimulated with leptin $(0.1-500 \mathrm{ng} / \mathrm{mL})$, EGF $(10 \mathrm{ng} / \mathrm{mL}), \mathrm{KGF}(10 \mathrm{ng} / \mathrm{mL})$, or a combination of the growth factors for an additional 24 hours. After growth-factor incubation, 5-bromo-2'-deoxyuridine (BrdU) labeling reagent (Roche Biochemicals), was added for an additional 1-hour incubation. BrdU incorporation during cellular S-phase, as assessed by BrdU colorimetric cell proliferation ELISA (Roche Biochemicals) was used as a direct readout for cell proliferation rates. Human recombinant leptin was purchased from R\&D Systems, and EGF and KGF were from Roche Biochemicals.

Proliferation assay. Keratinocytes ( $\mathrm{HaCaT}$ cell line and human primary cells) were seeded on 96-well plates. Each well contained $10^{3}$ cells in a total volume of 100 $\mu \mathrm{L}$ of DMEM $/ 10 \%$ FCS (for $\mathrm{HaCaT}$ ) or KBM-2 (for primary cells). Subsequently, cells were grown for 24 hours. The proliferation rate of incubated cells was determined after a 24-hour stimulation with leptin (100 ng/mL), KGF (10 ng/mL), EGF (10 ng/mL), or a combination of the growth factors using the CellTiter 96 Aqueous One Solution Cell Proliferation Assay (Promega, Mannheim, Germany), according to the instructions of the manufacturer. This assay determines the number of viable cells in proliferation assays, because the total amount of the formed formazan end product is directly proportional to the number of living cells in culture.

Electrophoretic mobility-shift assay. HaCaT keratinocytes (DMEM/10\% FCS) or human primary keratinocytes (KBM-2) were grown to $70 \%$ confluence and rendered quiescent by a 24-hour starvation in serum-free DMEM (for $\mathrm{HaCaT}$ ) or EGF-free KBM-2 (for primary cells). 
Subsequently, cells were stimulated with $100 \mathrm{ng} / \mathrm{mL}$ human recombinant leptin or $10 \mathrm{ng} / \mathrm{mL}$ EGF. After 10, 15,20 , or 30 minutes of incubation, cells were harvested for the preparation of nuclear extracts (26). Binding reactions were performed for 3 hours on ice using 10 $\mu \mathrm{g}$ of nuclear protein in $20 \mu \mathrm{L}$ of $4 \%$ Ficoll, $20 \mathrm{mM}$ HEPES, pH 7.9, $50 \mathrm{mM} \mathrm{KCl}, 1 \mathrm{mM}$ EDTA, 1 mM DTT, $1 \mathrm{mM}$ PMSF, $1 \mu \mathrm{g}$ poly dI/dC, and 20,000 cpm of ${ }^{32} \mathrm{P}-$ labeled STAT3 consensus oligo. DNA-protein complexes were separated from the free DNA probe by native polyacrylamide gel electrophoresis (4.5\%). Competition experiments were performed by coincubation of a 10-100-fold excess of unlabeled wild-type or mutated STAT3 consensus oligo, respectively. The wild-type and mutated STAT3 consensus oligos were from Santa Cruz Biotechnology Inc.

ELISA. Total protein $(50 \mu \mathrm{g}$ diluted in lysis buffer to a final volume of $50 \mu \mathrm{L}$ ) from nonwounded skin lysates and wound lysates, respectively, were subsequently analyzed for the presence of immunoreactive leptin protein by ELISA using the Quantikine murine leptin kit (R\&D Systems), as described by the manufacturer.

Statistical analysis. Data are shown as means plus or minus SD. Data were analyzed by unpaired Student's $t$ test on raw data using Sigma Plot (Jandel Scientific, Erkrath, Germany).

\section{Results}

Systemically and topically applied leptin reversed impaired wound healing in ob/ob mice. To determine a possible function of leptin for cutaneous wound healing, we investigated the effect of systemically and topically applied leptin for the healing process using a model of excisional skin repair in leptin-deficient $o b / o b$ mice. $O b / o b$ mice that were intraperitoneally supplemented with leptin (5 $\mu \mathrm{g} / \mathrm{g}$ body weight, once a day) were characterized by a markedly accelerated wound-healing process, compared with PBS-treated control mice. Whereas wounds in leptin-treated $o b / o b$ mice were completely re-epithelialized within 13 days, the wounds from PBS-treated control mice were still covered by a scab and not epithelialized at 13 days after wounding (Figure 1c). Histology demonstrated that wounds isolated from leptin-treated mice were covered by a well-organized epithelium, whereas wound re-epithelialization in PBS-treated control animals was remarkably retarded. Besides these effects, however, leptin decreased body weight and blood glucose levels in the animals (Figure 1a), clearly indicating that the recombinant murine leptin protein remained biologically active after systemic application. Because systemic supplementation of leptin in $o b / o b$ mice clearly reversed the diabetic phenotype of the animals, we had to evaluate the possibility that accelerated a
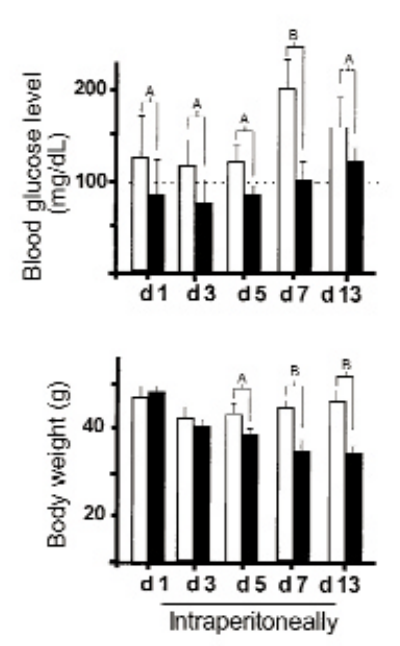

b
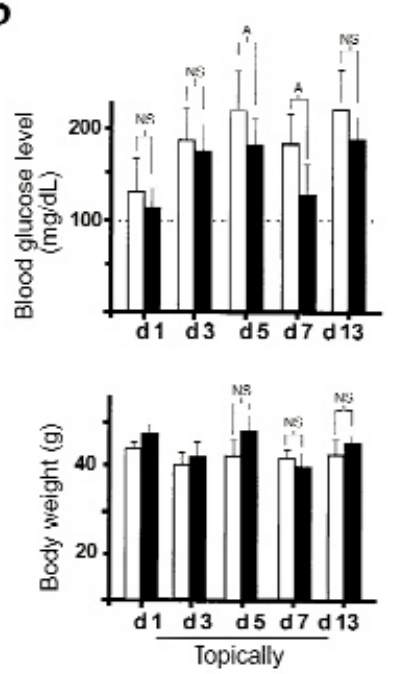

c

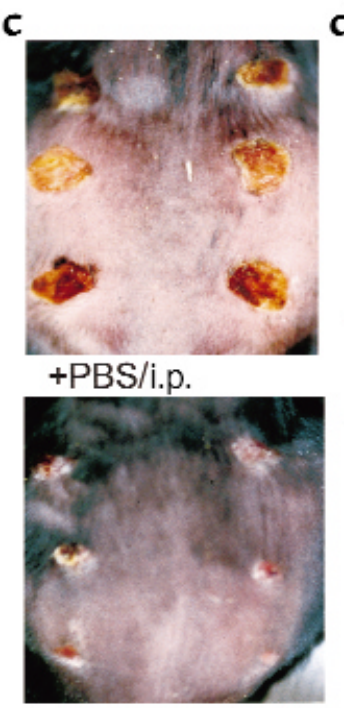

+leptin/i.p. d
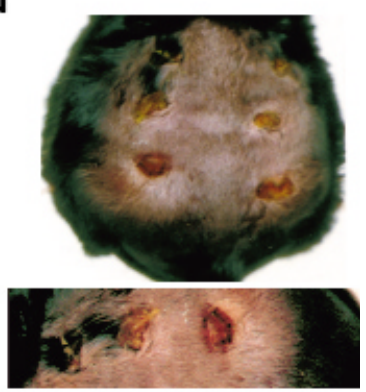

+PBS/top.

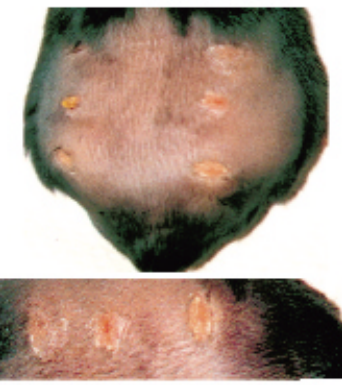

+leptin/top.

\section{Figure 1}

Effect of intraperitoneally and topically supplemented leptin on blood glucose level, body weight, and wound healing in ob/ob mice. C57BL/6J$o b / o b$ mice were treated with leptin intraperitoneally (i.p.) or topically (top) as described in Methods. PBS-treated $o b / o b$ mice were used as a control. ( $\mathbf{a}$ and $\mathbf{b}$ ) Blood glucose levels and body weight were determined for intraperitoneally injected (a) or topically treated (b) animals at the indicated experimental time points. Three animals $(n=3)$ were analyzed at every experimental time point. Data are expressed as milligrams per deciliter (for blood glucose levels) or grams (for body weight). Mean changes \pm SD in blood glucose levels, or body weight, respectively, are shown. ${ }^{A} P<0.05$; ${ }^{B} P<0.01$; NS, not significant compared with the conditions as indicated with the brackets. (c) Thirteen-day wounds after intraperitoneal (i.p.) treatment of animals with $5 \mu \mathrm{g}$ leptin/g of body weight (+leptin/i.p.) or PBS (+PBS/i.p.). (d) Back skin of representative C57BL/6J-ob/ob mice topically treated with $1 \mu \mathrm{g}$ leptin in $20 \mu \mathrm{L}$ PBS twice a day (+leptin/top.) or PBS (+PBS/top.) at day 10 after wounding. The bottom panels demonstrate the same wounds in higher magnification. 
re-epithelialization in leptin-treated mice was simply due to improvement of the diabetic phenotype rather than to a direct mitogenic effect of leptin on the epithelium. To this end, we investigated the effect of topically applied leptin on the wound-healing process. For this purpose wounds of $o b / o b$ mice were supplemented with $1 \mu \mathrm{g}$ leptin in $20 \mu \mathrm{L}$ PBS per wound twice a day. PBStreated animals were used as control. It is noteworthy that smallest amounts of topically applied leptin (1 $\mu \mathrm{g} /$ wound/day) turned out to be potent in stimulating repair. As shown in Figure 1d, topically applied leptin markedly improved wound repair, since the wounds of these mice had completely re-epithelialized at day 10 after wounding, whereas the wounds of PBS-treated control animals were still covered by a scab at this experimental time point. Moreover, we observed the same curative action of leptin in animals where the wounds located on the left side of the back were treated topically with leptin $(1 \mu \mathrm{g} /$ wound/day), whereas the wounds of the right side received vehicle solution only. This is demonstrated in Figure 2, a and d. After 10 days of leptin application, the left-side wounds, which had obtained the leptin-treatment, were characterized by a highly organized epithelium that covered the whole wounded area (Figure 2a). In contrast, the wounds located on the right sides of the same animals (treated with PBS vehicle only, Figure 2d) were not re-epithelialized, because the migrating epithelial tongues had not met to close the wound. Importantly, topically applied leptin only marginally reduced blood glucose levels,

\section{Figure 2}

Effect of leptin on epithelial proliferation and localization of leptinreceptor subtypes in the skin. (a and $\mathbf{d}$ ) Hematoxylin/eosin-stained frozen sections from 10-day wounds isolated from the same $\mathrm{C} 57 \mathrm{BL} / 6 \mathrm{~J}-o b / o b$ individual that had been treated topically with $1 \mu \mathrm{g}$ leptin/20 $\mu$ L PBS twice a day on the left-side wounds (a) or PBS only on the right-side wounds (d), respectively. Arrows indicate the leading edge of the migrating epithelium. ( $\mathbf{b}$ and $\mathbf{e}$ ) Frozen sections from 5-day wounds isolated from leptin-treated (intraperitoneally) (b) or PBS-injected (e) C57BL/6J-ob/ob mice were incubated with a monospecific, polyclonal Ab directed against murine Ki67. (c) Frozen section from a 13-day wound isolated from a leptin-treated (intraperitoneally) mouse. The section was incubated with a monospecific polyclonal $\mathrm{Ab}$ against the ObRb-receptor subtype. (f) Frozen section from a 7-day wound isolated from a PBS-treated (intraperitoneally) mouse. The section was incubated with a monospecific polyclonal $A b$ against the ObRb-receptor subtype. Note that the epithelium did not extend into the granulation tissue (compare to $\mathbf{b}$, epithelium of leptin-treated mouse, 5 days). (g) Frozen section from a 13-day wound isolated from a wild-type mouse $(B A L B / c)$. The section was incubated with a monospecific polyclonal Ab recognizing the ObRband ObRa-receptor subtypes. Sections were stained with the avidinbiotin-peroxidase complex system using 3-amino-9-ethylcarbazole as a chromogenic substrate $(\mathbf{b}, \mathbf{c}, \mathbf{e}, \mathbf{f}, \mathbf{g})$. Nuclei were counterstained with hematoxylin. Strongly immunopositive signals within the sections are indicated with arrows. e, epithelium; g, granulation tissue; he, hyperproliferative epithelium; s, scab. and, additionally, no changes in body weight were observed in mice treated topically with leptin (Figure 1b). These data strongly argue for a direct and local function of leptin for wound repair, and, moreover, the impaired wound healing observed in $o b / o b$ mice is only partially due to the metabolic changes caused by the diabetic phenotype.

Topically applied leptin accelerated wound healing in wild-type mice. Because systemically and topically supplemented leptin strongly improved the delayed wound closure in $o b / o b$ mice, we speculated that leptin might mediate a beneficial effect to normal wound-healing conditions. Thus, we treated wounds of wild-type mice (Balb/c) topically with $5 \mu \mathrm{g}$ leptin in $20 \mu \mathrm{L}$ PBS twice a day. PBStreated mice were used as a control. As shown in Figure 3a, we observed an accelerated closure of leptin-covered wounds. After 5 days of repair, leptin-treated wounds appeared to be markedly reduced in diameter and nearly completely re-epithelialized compared with PBS-treated wounds. Eosin/hematoxylin-stained sections of leptin- or PBS-treated wounds consistently revealed histological differences. After 5 days of leptin application, wounds were characterized by a dense neoepithelium that covered the whole wounded area (Figure 3b). By contrast, vehicle-treated (PBS) wounds developed hyperproliferative epithelia at the wound margins, but as it is well-known for this stage of a normal repair process in mice, the migrating epithelial tongues did not yet meet to completely cover the wound site. However, leptin function was most likely to be restricted to enhance

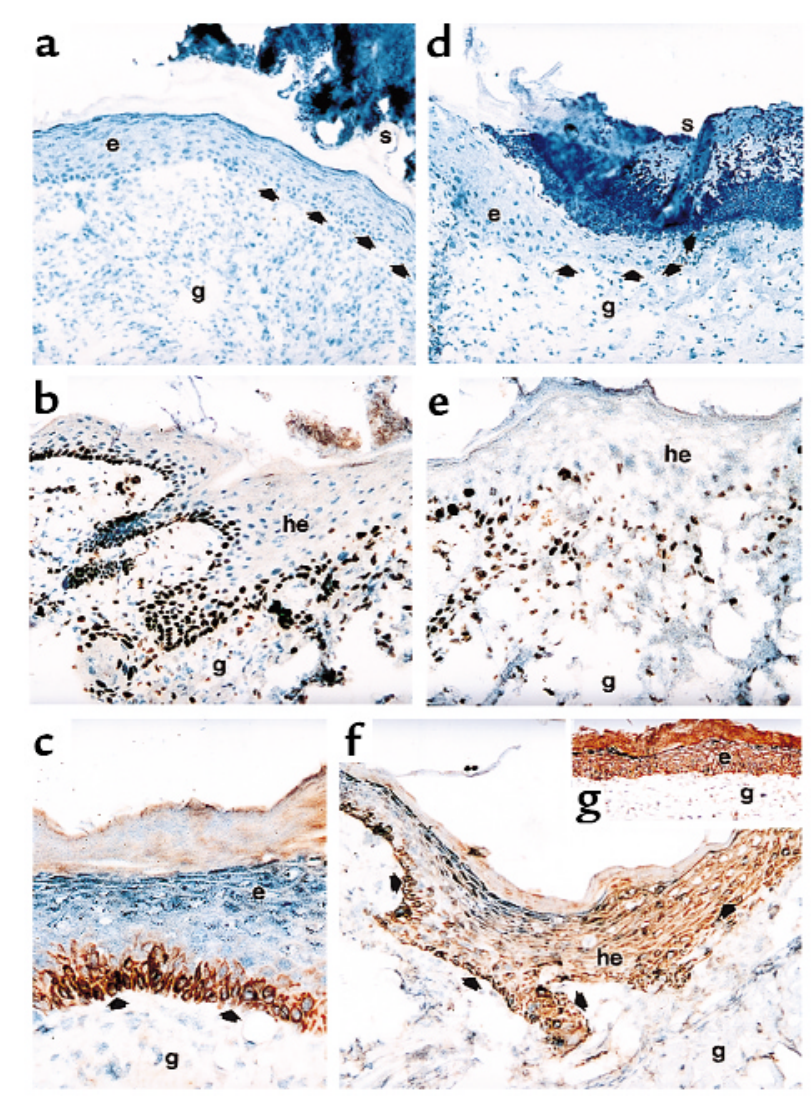



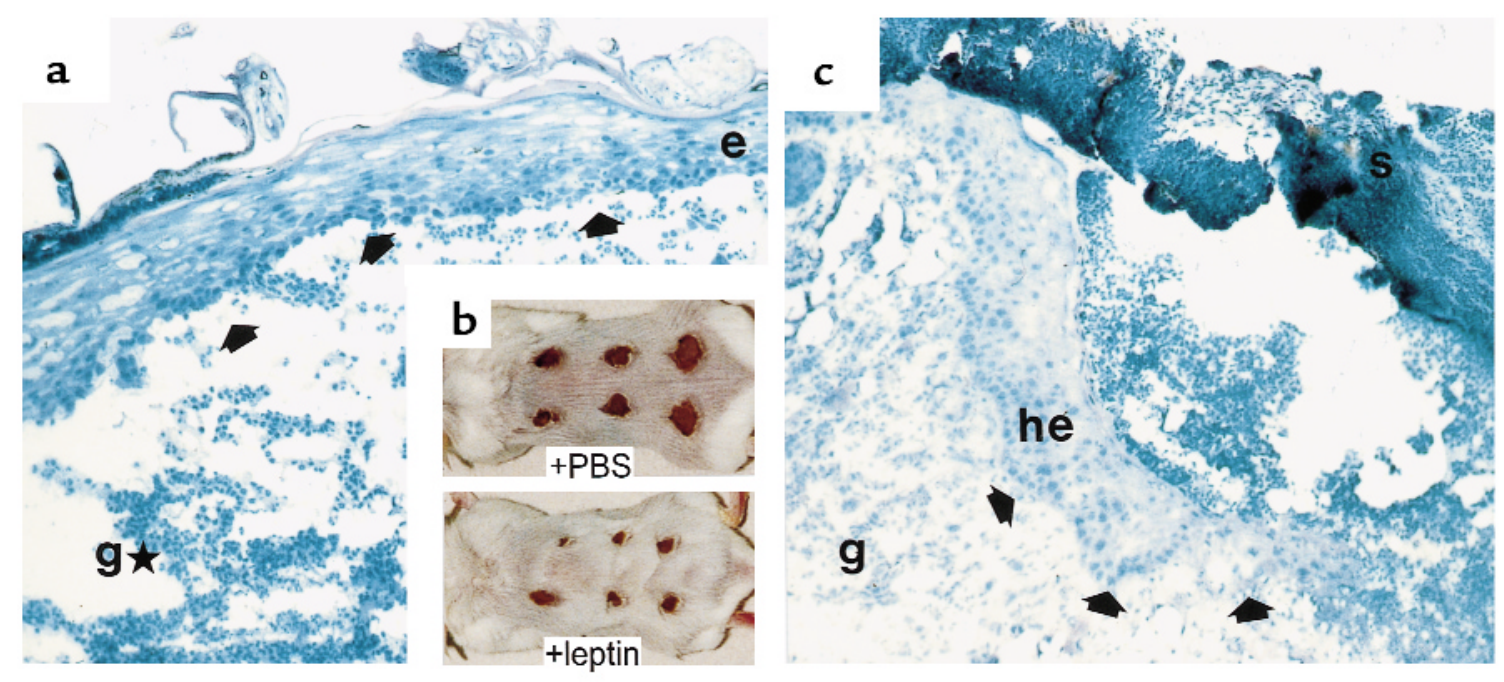

Figure 3

Effect of topically supplemented leptin on wound healing in wild-type mice. Balb/c mice were topically treated with leptin as described in Methods. PBS-treated mice were used as a control. (b) Wounded back skin of representative Balb/c mice ( 5 days after wounding). Wounds have been treated topically for the whole 5-day period after wounding with $5 \mu \mathrm{g}$ leptin/20 $\mu \mathrm{L}$ PBS twice a day (+leptin) or $20 \mu \mathrm{L}$ PBS (+PBS), respectively. (a and $\mathbf{c}$ ) Hematoxylin/eosin-stained frozen sections from 5-day wounds isolated from Balb/c mice that have been treated topically with $5 \mu \mathrm{g}$ leptin/20 $\mu \mathrm{L}$ PBS twice a day (a) or $20 \mu \mathrm{L}$ PBS only (c). Arrows indicate the leading edge of the migrating epithelium. e, epithelium; g, granulation tissue; $\mathrm{gH}$, atrophied granulation tissue; he, hyperproliferative epithelium; s, scab.

epithelial processes, because the underlying granulation tissue appeared to be characterized by a low cellularity and, hence, by deficiencies in the development of new stroma (Figure 3c). Thus, leptin function suggests a role primarily in triggering epithelial processes during repair.

The leptin-receptor isoforms ObRa and ObRb were differentially expressed during wound healing. Because topically applied leptin markedly enhanced wound re-epithelialization in $o b / o b$ and wild-type mice, we investigated the expression pattern of the leptin receptor in normal skin and during cutaneous repair in leptin- or PBS-injected (intraperitoneally) $o b / o b$ mice, respectively. Until now, four different splice variants of the leptin-receptor gene have been identified, from which only the ObRb isoform has been demonstrated to mediate intracellular signaling through activation of the Jak-STAT cascade (5). Using the RT-PCR technique, the ObRa and ObRb splice variants of the leptin receptor could be amplified from RNA of unwounded control skin and from 7-day or 13day wounds of wild-type mice $(\mathrm{Balb} / \mathrm{c})$ and $o b / o b$ mice. The identity has been confirmed by cloning and subsequent sequence analysis of the amplified receptor cDNA fragments (data not shown). To investigate regulation of ObR mRNA and protein expression during wound healing, we performed a RNase protection assay (Figure 4a) and a Western blot analysis (Figure 4b). We used an antisense RNA probe that hybridized to a mRNA region recognizing both leptin-receptor splice variants and a polyclonal $\mathrm{Ab}$ recognizing both ObRa and ObRb. As shown in Figure 4, ObR mRNA and protein were expressed in unwounded skin of $o b / o b$ mice, but wounding led to a rapid decline in ObR mRNA and protein levels. Minimal ObR expression was observed 3 days after wounding. ObR mRNA levels started to reincrease 5 days after injury, followed by clearly detectable levels of ObRb-specific proteins 7 days after wounding. It is noteworthy that the kinetics of ObR expression were identical for wild-type (Balb/c; data not shown) and leptin-supplemented $o b / o b$ mice (Figure 4, a and b). In these animals, interestingly, ObR mRNA levels remained elevated after re-epithelialization at day 13 compared with control skin. In contrast, PBS-treated $o b / o b$ mice did not show a marked recovery of ObR mRNA and protein expression after wounding, and ObR expression levels remained reduced even after 13 days of repair (Figure 4b, compare 13-day wound). Thus, we observed expression of the functional leptin receptor ObRb to be regulated during repair. Moreover, its ligand leptin was constitutively present in nonwounded skin and at the wound site during normal healing, because Balb/c control animals revealed leptin levels of approximately $30 \mathrm{pg} / 50 \mu \mathrm{g}$ total protein of nonwounded skin or total wound protein (day 1, 3, 5, 7 , and 13 after wounding), respectively, as assessed by leptin-specific ELISA. Therefore, leptin readjusted the dysregulated expression of its own receptor in leptindeficient $o b / o b$ mice and, furthermore, was most likely to trigger the enhanced expression of $\mathrm{ObRb}$ during the late phase of repair.

The leptin-receptor subtype ObRb is exclusively expressed in keratinocytes of the basal layer of the epidermis and the hyperproliferative epithelium at the wound edge. Because supplementation of leptin clearly accelerated wound re-epithelialization in $o b / o b$ mice, it was important to determine the potential target cells for leptin function at the wound site. Thus, we performed immunohistochemistry using antibodies specific for the ObRb-receptor subtype or both leptinreceptor subtypes, respectively. In control skin of 
wild-type (Balb/c) and ob/ob mice, ObRb protein was specifically expressed in keratinocytes of the basal layer of the epidermis (data not shown). As shown in Figure $2 \mathrm{c}$, the same ObRb expression pattern was observed in completely re-epithelialized wounds 13 days after injury. Moreover, ObRb expression was not restricted to the basal layer of the epidermis during repair, but strong immunopositive signals could be detected in keratinocytes of the migrating epithelial tongue at the wound margins (Figure 2f). This situation was found in wild-type and $o b / o b$ mice and turned out to be independent of the organization of the proliferative epithelium. In line with the data observed in Western blot analysis, a weak staining of keratinocytes of the hyperproliferative epithelium could be detected for the ObRb-receptor subtype at day 5 after injury (data not shown), but became clearly visible at day 7 after wounding (Figure 2f). ObRa protein was observed to be

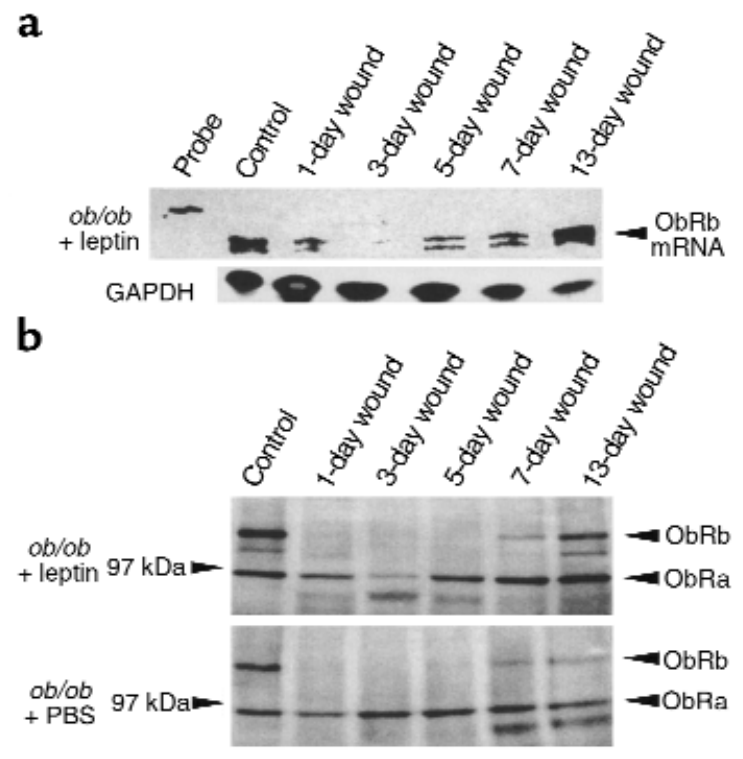

Figure 4

Regulation of leptin-receptor expression during wound healing in $\mathrm{Balb} / \mathrm{c}$ (wt) and C57BL/6J-ob/ob mice. (a) C57BL/6J mice were intraperitoneally treated with leptin as described in Methods. Total cellular RNA $(20 \mu \mathrm{g})$ from nonwounded and wounded back skin of C57BL/6J-ob/ob mice treated with leptin as indicated was analyzed by RNase protection assay with an RNA hybridization probe complementary to ObRb and ObRa leptin-receptor splice variants. For every experimental time point, three wounds each from three animals (total: $n=9$ wounds) were pooled for analysis. The time after injury is indicated for each lane. Control skin refers to nonwounded skin. Hybridization probe (1000 counts/min) was added to the lane labeled probe. Expression of GAPDH mRNA is shown as a loading control in the bottom panel. (b) Total protein $(50 \mu \mathrm{g})$ from lysates of nonwounded and wounded back skin (day $1,3,5,7$, and 13 after injury, indicated for each lane) of C57BL/6J-ob/ob mice treated with leptin or PBS (used as control) as indicated were analyzed by immunoblotting for the presence of leptin-receptor subtype proteins ObRa and ObRb. Two wounds from the backs of three animals $(n=6)$ were excised for each experimental time point and used for protein isolation. Leptinreceptor subtypes ObRa and $\mathrm{ObRb}$ are indicated by arrowheads. expressed specifically in keratinocytes of wild-type and $o b / o b$ mice. In contrast to ObRb isoform expression, ObRa staining was detected throughout all layers of the epidermis (Figure $2 \mathrm{~g}$ ).

Because expression of the ObRb leptin-receptor isoform was restricted to proliferating keratinocytes (epidermal basal layer, hyperproliferative epithelium), we hypothesized that leptin might mediate a mitogenic stimulus to keratinocytes located at the wound margins. Therefore, we investigated the proliferation pattern within the hyperproliferative epithelia of leptin-injected or PBS-injected $o b / o b$ mice, respectively. For this purpose, we stained sections from leptin-treated or PBS-treated $o b / o b$ mice with an $\mathrm{Ab}$ detecting the proliferation marker Ki67 (27). Note that the epithelial tongues from wounds of PBS-injected control mice were characterized by a disorganized and atrophied morphology and, furthermore, a diffuse distribution of Ki67-positive, proliferating keratinocytes (Figure 2e). In contrast, wounds from leptin-injected mice showed a highly organized and massive bulk of keratinocytes at the wound margins. These wounds were characterized not only by hyperproliferative epithelia that covered the wound, but, additionally, by Ki67-positive keratinocytes that reached out deep into the granulation tissue (Figure 2b). Moreover, the total number of keratinocytes and Ki67-positive proliferating keratinocytes appeared to be markedly reduced in wounds isolated from leptin-deficient, PBS-treated control animals (Figure 5a). These results suggest that leptin might function as a mitogenic factor to keratinocytes during repair, probably by acting on the leptin-receptor isoform ObRb, which is exclusively expressed in this cell type within the skin.

Leptin-enhanced proliferation and activated STAT3 in the buman keratinocyte cell line $\mathrm{HaCaT}$ and human primary keratinocytes. To further strengthen our in vivo observations, we determined the mitogenic potency of leptin in keratinocytes in vitro. First, by RT-PCR amplification and subsequent cloning and sequencing of the amplified cDNA fragments, we assessed that the human keratinocyte cell line HaCaT (25) and, additionally, human primary keratinocytes indeed expressed the ObRb splice variant, suggesting that the cells are susceptible to leptin-mediated signaling (data not shown). Using the BrdU cell-proliferation ELISA, we observed a strong and dose-dependent proproliferative effect of recombinant human leptin on the $\mathrm{HaCaT}$ cell line (Figure 5b, shaded bars) and the human primary keratinocytes (Figure 5b, filled bars). Remarkably, the mitogenic effects could be observed even at the lowest leptin concentrations $(0.1 \mathrm{ng} / \mathrm{mL})$ used. Note that the medium used for culturing human primary keratinocytes (KBM-2; see Methods) included epinephrine $(0.5 \mu \mathrm{g} / \mathrm{mL})$, which is known to increase the activity of adenylate cyclase (28) and, subsequently, cAMP concentrations (29) in keratinocytes. The proproliferative effect of leptin was comparable to the effects observed by the well-known epithelial mitogens EGF and KGF (Figure 5c, left panel). Furthermore, 


\section{Figure 5}

Effect of leptin on proliferation and STAT3 activation in $\mathrm{HaCaT}$ keratinocytes and human primary keratinocytes. (a) Low-magnification photographs $(100 \times)$ of frozen serial sections were analyzed for total keratinocyte cell numbers or proliferating keratinocytes within the hyperproliferative epithelia from leptintreated (intraperitoneally) $a b / o b$ mice and PBS-treated (intraperitoneally) ob/ob mice $(n=3)$, as indicated. This was done by counting hematoxylin-stained keratinocyte nuclei or Ki67-immunostained keratinocyte nuclei in a defined area $(3 \times$ $2 \mathrm{~mm}$ ). Data are expressed as total number of keratinocyte nuclei $\pm \mathrm{SD}(n=3) .{ }^{A} P<0.01$ as indicated by the brackets, percentage of means compared with leptin-treated animals. (b) Dose-dependent effects of human recombinant leptin (0.1-500 $\mathrm{ng} / \mathrm{mL}$ ) on $\mathrm{HaCaT}$ (open bars) or primary (filled bars) keratinocyte proliferation. Proliferation was determined by BrdU incorporation. Data are expressed as percentage of unstimulated control. Mean percentage of change in proliferation \pm SD are shown (values represent the mean of five assays with a readout of nine wells for each concentration; $n=45)$. ${ }^{A} P<0.01 \mathrm{com}$ pared with control; $\mathrm{B} P<0.05$ compared with control. (c) Effects of leptin $(100 \mathrm{ng} / \mathrm{mL}), \operatorname{KGF}(10 \mathrm{ng} / \mathrm{mL})$, or EGF $(10 \mathrm{ng} / \mathrm{mL})$ on $\mathrm{HaCaT}$ (open bars) and primary (filled bars) keratinocyte proliferation. KGF and leptin or EGF and leptin were also given simultaneously, as indicated. Proliferation was determined by BrdU incorporation (left panel) or using the MTS reagent (right panel) as described in Methods. Data are expressed as percentage of unstimulated control. Mean percentage of change in proliferation \pm SD are shown (values represent the mean of six assays with a readout of nine wells for each condition; $n=$ 54). ${ }^{A} P<0.01$ compared with control. (d and e) EMSA. HaCaT keratinocytes (d) and primary keratinocytes (e) were grown to confluence and rendered quiescent by a 24-hour incubation in serum-free medium. Cells were subsequently stimulated with leptin $(100 \mathrm{ng} / \mathrm{mL})$ or EGF $(10 \mathrm{ng} / \mathrm{mL})$ for the indicated time periods. Nuclear extracts of stimulated cells were isolated as described in Methods. Specificity of binding was confirmed by competition experiments using a 10-100-fold excess of unlabeled wild-type (STAT3 $\mathrm{wt}$ ) or mutated STAT3 (STAT3 mut) consensus oligo as indicated. Lep, leptin; KC, keratinocyte. a

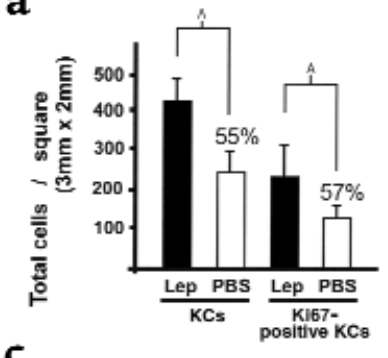

b

C

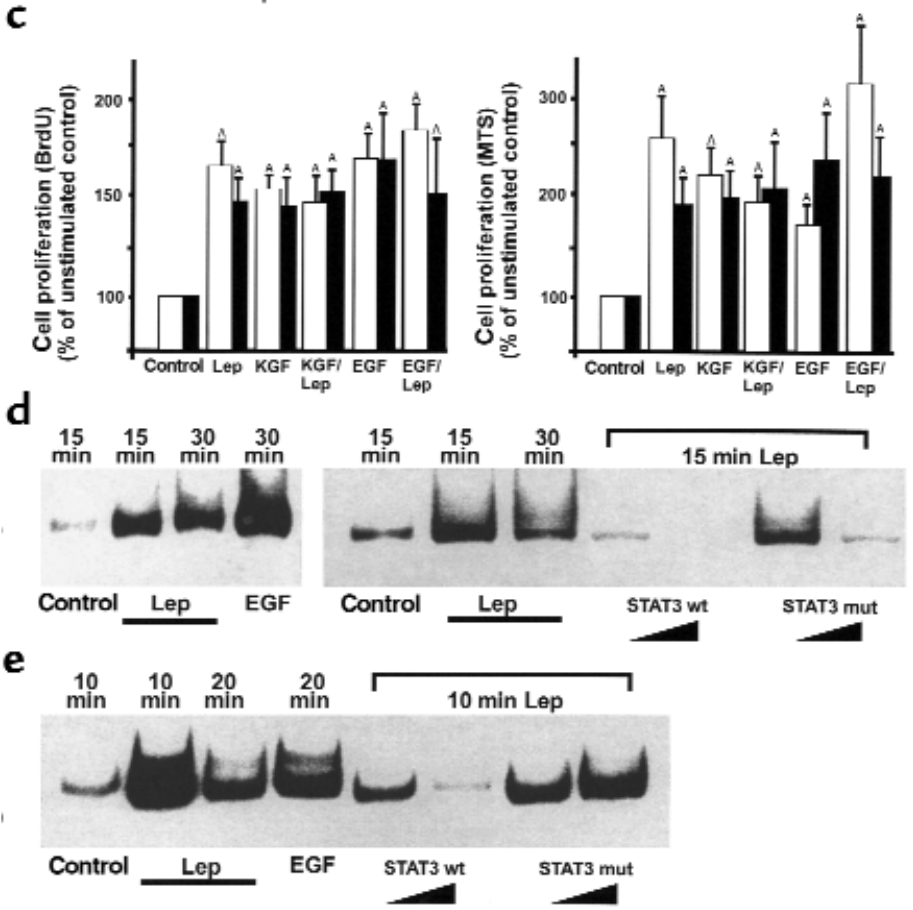

these results were confirmed using the MTS assay (Figure $5 \mathrm{c}$, right panel), which represents a colorimetric method to determine the number of viable cells in proliferation assays. Additionally, the mitogenic effects of leptin observed in the human keratinocyte cell line and human primary keratinocytes could be seen in murine primary keratinocytes (data not shown).

These results implicated a signaling machinery in keratinocytes that could respond to a leptin-mediated stimulus. Since it has been described that binding of leptin to its receptor activates Jak tyrosine kinases, which then phosphorylate STATs (5), we investigated whether a similar signal-transduction pathway is operative in keratinocytes. Therefore, we determined the potency of leptin to activate the transcription factor STAT3 in the keratinocyte cell line HaCaT (Figure 5d) and primary keratinocytes (Figure 5e), respectively, because nuclear translocation of STAT3 has been described as a result of ObRb-receptor activation in the hypothalamus or endothelial cells $(4,17)$. Using the electrophoretic mobility-shift assay (EMSA) technique, we observed a rapid and strong increase in activated
STAT3 within 15 minutes of leptin stimulation. EGFmediated STAT3 activation was used as a positive control (30), and specificity of the STAT3 complex was confirmed by competition experiments using mutated or wild-type STAT3 consensus oligonucleotides, respectively (Figure 5, d and e).

\section{Discussion}

For many years, $a b / a b$ mice have been used as a model system for impaired wound healing, and, additionally, the observed wound-healing disorders have been explained by the diabetic phenotype of these animals (31). However, it is well-known that the genetic background C57/BL6J gives rise to a moderate diabetic phenotype that occurs only transiently and predominantly in early developmental stages (32), indicating that moderate hyperglycemia might not represent the only reason for the impaired wound healing observed in leptin-deficient animals. The present study demonstrates that impaired repair in $o b / o b$ mice might result from a lack of leptin acting as a mitogenic factor for keratinocytes at the wound site. Beneficial effects of leptin 
were not restricted to leptin-deficient mice, because leptin markedly promoted the re-epithelialization processes in wild-type mice. Our data provide direct evidence that the beneficial effect of leptin on wound repair is due to a direct mitogenic action of leptin on keratinocytes located at the wound margins. Growth factors driving re-epithelialization are central to the wound-healing process. Crucial roles for this process have been elucidated for KGF, EGF, and TGF- $\alpha$, which have been shown to stimulate re-epithelialization in animal models $(33,34)$ or to be absent in models of impaired re-epithelialization (35). In line with these observations, keratinocytes of the hyperproliferative epithelium at the wound edge are known to express the KGF- or EGF-receptor $(20,36)$, respectively. According to these observations, we found a comparable expression pattern for the ObRb leptin-receptor isoform that is expressed in keratinocytes of the hyperproliferative epithelium. These keratinocytes are known to be undifferentiated and still able to proliferate (37).

In contrast to the observed constitutive expression of the KGF receptor (23) or elevated expression of the EGF receptor (38) in wound repair, the ObRb leptinreceptor expression is downregulated early after injury. This regulation might reflect an adaptive mechanism to counteract the effect of an immediately available leptin at the wound site. Whereas KGF or EGF are released in high amounts upon injury from fibroblasts or platelets (18), respectively, and subsequently trigger a rapid epithelial response, the downregulation of ObRb after injury is most likely to target specifically the mitogenic properties of leptin to later epithelial movements during repair. Among the four identified leptin-receptor subtypes, the ObRb form is characterized by a long cytoplasmic region with consensus sequences for the binding of Jak tyrosine kinases and binding of the transcription factor STAT3. Thus, the ObRb-receptor subtype has been shown to be responsible for leptin-dependent signaling in the hypothalamus. The other known leptinreceptor subtypes carry truncated cytoplasmic regions and, presumably, are not able to activate the Jak-STAT pathway. Because we identified the ObRb-receptor subtype to be specifically expressed in the proliferating keratinocytes of the epidermal basal layer in nonwounded skin and the hyperproliferative epithelia during repair, it is tempting to argue that the ObRbreceptor subtype system might serve to target the mitogenic effect of leptin toward the epithelial compartment. This assumption is further supported by the observation that $d b / d b$ mice, which are deficient in ObRb signaling but not for the other known leptinreceptor splice variants, including ObRa, are characterized by severe wound-healing disorders (39).

The observation that leptin promoted the proliferation of keratinocytes is in accordance with results obtained from various cell types because leptin reportedly has the potency to mediate mitogenic effects on lung epithelial cells, hemopoietic cells, pancreatic beta cells, monocytes, or endothelial cells $(11-16,40)$. However, this is the first report, to our knowledge, that demonstrates a proliferative function of leptin for an in vivo situation.

Until now, only little information dealing with the intracellular cascade involved in leptin-dependent signaling has been available. The leptin-receptor subtype ObRb has considerable homology to class I cytokine receptors (24). Several studies have demonstrated that binding of leptin causes receptor dimerization with association and activation of Jak kinases. Subsequently, STAT proteins are recruited to this complex and phosphorylated $(4,5)$. In line with these observations, it has been shown for lung epithelial cells that leptin mediates its mitogenic effects through the $\mathrm{ObRb}$ subtype (41). Accordingly, our data demonstrate that leptin activated STAT3 in keratinocytes. In agreement with these results, keratinocyte-specific STAT3-deficient mice were characterized by a severely impaired wound repair (42). In these animals, STAT3 deficiency was targeted to keratinocytes using a keratin-5 promoter construct. Thus, these data clearly underline an important role for a leptin-stimulated ObRb receptor-signaling cascade during repair, which subsequently mediates STAT3 activation in keratinocytes.

In summary, the possibility of an impairment of leptin-triggered epithelial processes must be considered to be crucially involved in the wound-healing deficiencies observed in $o b / o b$ mice. More important, our data provide evidence for beneficial effects of topically applied leptin for normal wound-healing situations. In this study we have identified leptin as a potent mediator of keratinocyte proliferation during wound healing in vivo. Since the absence of leptin from the wound is accompanied by an impaired re-epithelialization, we must consider leptin, besides the well-known mitogens, as a novel therapeutic factor to improve severely disturbed wound-healing conditions.

\section{Acknowledgments}

This work was supported by a grant of the Deutsche Forschungsgemeinschaft (SFB 553) and by the Paul and Ursula Klein Stiftung.

\footnotetext{
1. Zhang, Y., et al. 1994. Positional cloning of the mouse obese gene and its human homologue. Nature. 372:425-432.

2. Masuzaki, H., et al. 1995. Human obese gene expression. Adipocytespecific expression and regional differences in the adipose tissue. Diabetes. 44:855-858.

3. Halaas, J.L., et al. 1995. Weight-reducing effects of the plasma protein encoded by the obese gene. Science. 269:543-546.

4. Vaisse, C., et al. 1996. Leptin activation of STAT3 in the hypothalamus of wild-type and $o b / o b$ mice but not $d b / d b$ mice. Nat. Genet. 14:95-97.

5. Ghilardi, N., et al. 1996. Defective STAT signaling by the leptin receptor in diabetic mice. Proc. Natl. Acad. Sci. USA. 93:6231-6235.

6. Fei, H., et al. 1997. Anatomic localization of alternatively spliced leptin receptors (Ob-R) in mouse brain and other tissues. Proc. Natl. Acad. Sci. USA. 94:7001-7005.

7. Lollmann, B., Gruninger, S., Stricker-Krongrad, A., and Chiesi, M. 1997. Detection and quantification of the leptin receptor splice variants Ob-Ra, b, and e in different mouse tissues. Biochem. Biophys. Res. Commun. 238:648-652.

8. Hoggard, N., et al. 1997. Localization of leptin receptor mRNA splice variants in murine peripheral tissues by RT-PCR and in situ hybridiza-
} 
tion. Biochem. Biophys. Res. Commun. 232:383-387.

9. Chehab, F.F., Lim, M.E., and Lu, R. 1996. Correction of the sterility defect in homozygous obese female mice by treatment with the human recombinant leptin. Nat. Genet. 12:318-320.

10. Chehab, F.F., Mounzih, K., Lu, R., and Lim, M.E. 1997. Early onset of reproductive function in normal female mice treated with leptin. Science. 275:88-90.

11. Bouloumie, A., Drexler, H.C., Lafontan, M., and Busse, R. 1998. Leptin, the product of $\mathrm{Ob}$ gene, promotes angiogenesis. Circ. Res. 83:1059-1066

12. Santos-Alvarez, J., Goberna, R., and Sanchez-Margalet, V. 1999 Human leptin stimulates proliferation and activation of human circulating monocytes. Cell. Immunol. 194:6-11.

13. Tsuchiya, T., Shimizu, H., Horie, T., and Mori, M. 1999. Expression of leptin receptor in lung: leptin as a growth factor. Eur. J. Pharmacol. 365:273-279.

14. Tanabe, K., Okuya, S., Tanizawa, Y., Matsutani, A., and Oka, Y. 1997. Leptin induces proliferation of pancreatic beta cell line MIN6 through activation of mitogen-activated protein kinase. Biochem. Biophys. Res. Commun. 241:765-768.

15. Islam, M.S., Morton, N.M., Hansson, A., and Emilsson, V. 1997. Rat insulinoma-derived pancreatic beta-cells express a functional leptin receptor that mediates a proliferative response. Biochem. Biophys. Res. Commun. 238:851-855.

16. Konpleva, M., et al. 1999. Expression and function of leptin receptor isoforms in myeloid leukemia and myelodysplastic syndromes: proliferative and anti-apoptotic activities. Blood. 93:1668-1676.

17. Sierra-Honigmann, M.R., et al. 1998. Biological action of leptin as an angiogenic factor. Science. 281:1683-1686.

18. Martin, P. 1997. Wound healing: aiming for perfect skin regeneration. Science. 276:75-81.

19. Nanney, L.B. 1990. Epidermal and dermal effects of epidermal growth factor during wound repair. J. Invest. Dermatol. 94:624-629.

20. Marchese, C., et al. 1995. Modulation of keratinocyte growth factor and its receptor in reepithelializing human skin. J. Exp. Med. 182:1369-1376.

21. Frank, S., Stallmeyer, B., Kämpfer, H., Kolb, N., and Pfeilschifter, J. 1999. Nitric oxide triggers enhanced induction of vascular endothelial growth factor expression in cultured keratinocytes (HaCaT) and during cutaneous wound repair. FASEB J. 13:2002-2014.

22. Chomczynski, P., and Sacchi, N. 1987. Single-step method of RNA isolation by acid guanidinium thiocyanate-phenol-chloroform extraction. Anal. Biochem. 162:156-159.

23. Werner, S., et al. 1992. Large induction of keratinocyte growth factor expression in the dermis during wound healing. Proc. Natl. Acad. Sci. USA. 89:6896-6900.

24. Tartaglia, L.A., et al. 1995. Identification and expression cloning of a leptin receptor, OB-R. Cell. 83:1263-1271.
25. Boukamp, P., et al. 1988. Normal keratinization in a spontaneously immortalized aneuploid human keratinocyte cell line. J. Cell Biol. 106:761-771.

26. Schreiber, E., Matthias, P., Müller, M.M., and Schaffner, W. 1989. Rapid detection of octamer binding proteins with "mini-extracts" prepared from a small number of cells. Nucl. Acids Res. 17:6419.

27. Gerdes, J., et al. 1984. Cell cycle analysis of a cell proliferation associated human nuclear antigen defined by the monoclonal antibody Ki67. J. Immunol. 133:1710-1715.

28. Cavey, M.T., Cavey, D., Shroot, B., Reichert, U., and Gazith, J. 1986. Receptor-linked adenylate cyclase in the membranes of cultured human epidermal keratinocytes. Arch. Dermatol. Res. 278:293-297.

29. Takahashi, H., Tamura, T., Tsutsui, M., and Iizuka, H. 1990. Adenylate cyclase in fetal rat keratinizing epidermal cells (FRSK cells) and SV40transformed human keratinocytes. J. Dermatol. 17:457-464.

30. Darnell, J.E.,Jr. 1997. STATS and gene regulation. Science. 277:1630-1635.

31. Klingbeil, C.K., Cesar, L.B., and Fiddes, J.C. 1991. Basic fibroblast growth factor accelerates tissue repair in models of impaired wound healing. Prog. Clin. Biol. Res. 365:443-458

32. Coleman, D.L. 1982. Diabetes: obesity syndromes in mice. Diabetes. 31:1-6.

33. Brown, G.L., et al. 1989. Enhancement of wound healing by topical treatment with epidermal growth factor. N. Engl. J. Med. 321:76-79.

34. Schultz, G.S., et al. 1987. Epithelial wound healing enhanced by transforming growth factor-alpha and vaccinia growth factor. Science. 235:350-352.

35. Werner, S., et al. 1994. The function of KGF in morphogenesis of epithelium and reepithelialization of wounds. Science. 266:819-822.

36. Wenczak, B.A., Lynch, J.B., and Nanney, L.B. 1992. Epidermal growth factor receptor distribution in burn wounds. Implications for growth factor-mediated repair. J. Clin. Invest. 90:2392-2401.

37. Mansbridge, J.N., and Knapp, A.M. 1987. Changes in keratinocyte maturation during wound healing. J. Invest. Dermatol. 89:253-263.

38. Stoscheck, C.M., Nanney, L.B., and King, L.E. 1992. Quantitative determination of EGF-R during epidermal wound healing. J. Invest. Dermatol. 99:645-649.

39. Tsuboi, R., Shi, C.M., Rifkin, D.B., and Ogawa, H. 1992. A wound healing model using healing-impaired diabetic mice. J. Dermatol. 19:673-675.

40. Gainsford, T., et al. 1996. Leptin can induce proliferation, differentiation, and functional activation of hemopoietic cells. Proc. Natl. Acad. Sci. USA. 93:14564-14568.

41. Takahashi, Y., et al. 1997. Leptin induces mitogen-activated protein kinase-dependent proliferation of C3H10T1/2 cells. J. Biol. Chem. 272:12897-12900.

42. Sano, S., et al. 1999. Keratinocyte-specific ablation of STAT3 exhibits impaired skin remodeling, but does not affect skin morphogenesis. EMBO J. 18:4657-4668. 